\title{
Fiber-based, trace-gas, laser transmitter technology development for space
}

\author{
Mark Stephen ${ }^{* a}$, Anthony Yu ${ }^{a}$, Jeffrey Chen ${ }^{a}$, Jeffrey Nicholson ${ }^{b}$, Doruk Engin ${ }^{c}$, Brian Mathason ${ }^{c}$, \\ Stewart $\mathrm{Wu}^{\mathrm{a}}$, Graham Allan ${ }^{\mathrm{d}}$, William Hasselbrack ${ }^{\mathrm{d}}$, Brayler Gonzales ${ }^{\mathrm{a}}$, Lawrence Han ${ }^{\mathrm{a}}$, Kenji \\ Numata $^{\mathrm{e}}$, Mark Storm ${ }^{\mathrm{c}}$, James Abshire ${ }^{\mathrm{a}}$

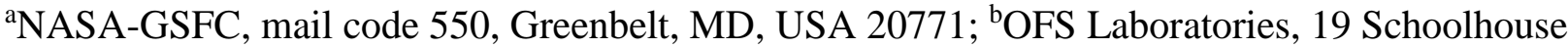

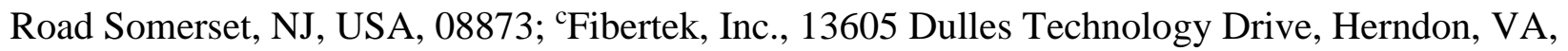 \\ USA, 20171; d Sigma Space Corporation, 4600 Forbes Blvd, Lanham-Seabrook, MD, USA, 20706; \\ eUniversity of Maryland, College Park, MD, USA, 20742
}

\begin{abstract}
NASA's Goddard Space Flight Center (GSFC) is working on maturing the technology readiness of a laser transmitter designed for use in atmospheric $\mathrm{CO}_{2}$ remote-sensing. GSFC has been developing an airplane-based $\mathrm{CO}_{2}$ lidar instrument over several years to demonstrate the efficacy of the instrumentation and measurement technique and to link the science models to the instrument performance. The ultimate goal is to make space-based satellite measurements with global coverage. In order to accomplish this, we must demonstrate the technology readiness and performance of the components as well as demonstrate the required power-scaling to make the link with the required signal-to-noise-ratio (SNR). To date, all the instrument components have been shown to have the required performance with the exception of the laser transmitter.

In this program we are working on a fiber-based master oscillator power amplifier (MOPA) laser transmitter architecture where we will develop a ruggedized package and perform the relevant environmental tests to demonstrate TRL-6. In this paper we will review our transmitter architecture and progress on the performance and packaging of the laser transmitter.
\end{abstract}

Keywords: fiber laser, lidar, IPDA, remote sensing, space, EDFA

\section{INTRODUCTION}

NASA's Goddard Space Flight Center (GSFC) is working on maturing the technology readiness of a laser transmitter designed for use in atmospheric carbon dioxide $\left(\mathrm{CO}_{2}\right)$ satellite remote-sensing. GSFC has been developing an airplanebased $\mathrm{CO}_{2}$ lidar instrument over several years to demonstrate the efficacy of the instrumentation and measurement technique and to link the science models to the instrument performance. The ultimate goal is to make space-based satellite measurements with global coverage. In order to accomplish this, we must demonstrate the technology readiness and performance of the components as well as demonstrate the required power-scaling to make the link with the required signal-to-noise-ratio (SNR).

In this program we are working on a fiber-based MOPA laser transmitter architecture where we will develop a ruggedized package and perform the relevant environmental tests to demonstrate technology readiness level (TRL) to 6. In this paper we will review our transmitter architecture and progress on the performance and packaging of the laser transmitter.

Our technical objective is to increase the TRL of the pulsed $1.57 \mu \mathrm{m}$ fiber-based laser for the $\mathrm{CO}_{2}$ Sounder to TRL-6. We will demonstrate the key performance requirements for a space-based $\mathrm{CO}_{2}$ sounder laser transmitter and complete environmental testing of the laser transmitter. This will remove the last technology hurdle to enable active $\mathrm{CO}_{2}$ measurements from space. It will also mature high power fiber-laser technology for other NASA applications.

\section{DESIGN AND PERFORMANCE}

Our pulsed laser transmitter is rapidly step-tuned across an atmospheric $\mathrm{CO}_{2}$ absorption line centered at $1572.335 \mathrm{~nm}$. We continue to evaluate the optimum number of wavelengths [1] required to scan the line (but we assume 8 wavelength steps

*mark.a.stephen@nasa.gov; phone 1301 614-6737; 
here.) The seed lasers are rapidly switched from fixed locked wavelength points, producing a repeating wavelength-stepped pulse train. The lasers cycle through all wavelength steps every $\sim 1 \mathrm{msec}$. The pulses are separated by $\sim 130 \mu \mathrm{sec}$, (the time required to completely clear the bottom $19 \mathrm{~km}$ of the atmosphere before the next pulse enters, eliminating crosstalk from cloud scattering.) This corresponds to a pulse repetition rate of $7.5 \mathrm{kHz}$. Stepping across the absorption lines in wavelength allows margin to correct for any drifts or non-uniformity in the line spectroscopy and in the lidar wavelength response. At a spacecraft velocity of $7 \mathrm{~km} / \mathrm{sec}$ the $\sim 100 \mathrm{~m}$ diameter laser spot advances $\sim 7 \mathrm{~m}$ for each 1-ms wavelength scan. In a 10 second averaging time, the highly overlapped sampling minimizes any errors caused by variability in surface reflectivity.

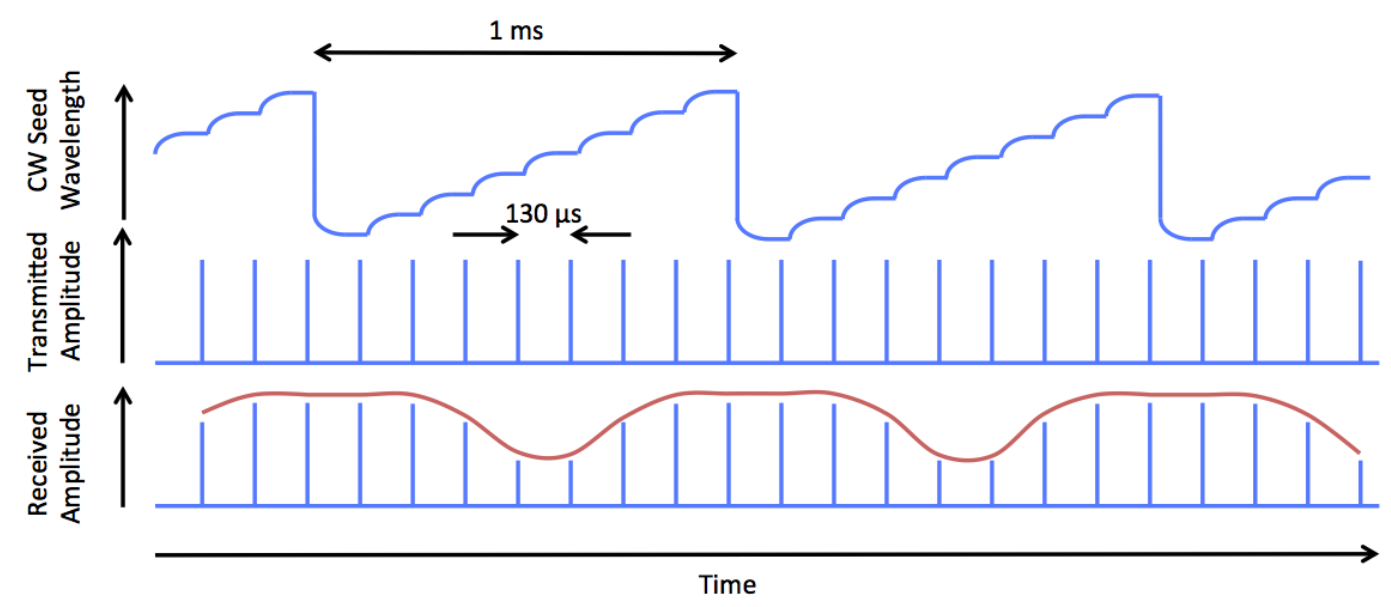

Figure 1. Wavelength, Amplitude Timing Diagram. The top shows stepped wavelength cycle of 8 different wavelengths. The middle graph shows the $1 \mu$ s pulses after external modulation (corresponding in time to after the wavelength has been locked) and the bottom graph shows the received signal after the $\mathrm{CO}_{2}$ absorption. The red curve shows the resultant absorption profile. The time between adjacent wavelengths and pulses is $130 \mu \mathrm{s}$, the time for one wavelength sweep is 1 ms.

A summary of the optical performance requirements is shown in Table 1. These requirements are derived from the science goals of the $\mathrm{CO}_{2}$ measurement [2] and the instrument architecture [3]. We decided on a Master Oscillator Power Amplifier (MOPA) laser architecture due to the complicated spectral requirements and decided on erbium-doped fiber amplifiers (EDFA) to achieve the desired power scaling due to the maturity of the technology and previously demonstrated performance [4-6].

Table 1. Laser Transmitter Optical Performance Requirements.

\begin{tabular}{ll} 
Performance Parameter & 8-Channel Combined Transmitter \\
\hline Center Wavelength & Nominally centered at $1572.335 \mathrm{~nm}$ \\
\hline
\end{tabular}




\begin{tabular}{|c|c|}
\hline Wavelength Span & 1572.23 to 1572.43 (in 8 wavelength steps, TBR) \\
\hline Tuning speed & $\sim 100 \mu \mathrm{s}$ \\
\hline Linewidth (each channel) & $\leq 100 \mathrm{MHz}$ \\
\hline Side-mode suppression ratio (spectral) & $>30 \mathrm{~dB}$ \\
\hline Wavelength stability (each channel) fast & Locked to $<3 \mathrm{MHz}$ \\
\hline $\begin{array}{l}\text { Wavelength stability (each channel) } \\
\text { slow }\end{array}$ & Locked to $<0.3 \mathrm{MHz}$ \\
\hline Pulse repetition frequency & $7.5 \mathrm{KHz}$ \\
\hline Pulse period & $133 \mu \mathrm{s}$ \\
\hline Pulse Width & $1 \mu \mathrm{s}$ \\
\hline Duty Cycle & $0.75 \%$ \\
\hline Pulse energy & $\begin{array}{l}\text { Sum at Farfield: }>3.2 \mathrm{~mJ} / \text { pulse (goal), } \\
>2.5 \mathrm{~mJ} / \text { pulse (operating, } 18 \% \text { derating) }\end{array}$ \\
\hline Average optical power & Sum at Far Field: >24 W (goal); $20 \mathrm{~W}$ (op) \\
\hline Peak power & $3.2 \mathrm{KW}$ goal, $2.5 \mathrm{~kW}$ operating \\
\hline Pulse Extinction ratio (timing) & $>30 \mathrm{~dB}$ \\
\hline$\%$ of power in the pulses (derived) & $90 \%$ \\
\hline ASE & $<1 \%$ of average power \\
\hline Margin to SBS threshold & $>2 \mathrm{X}$ \\
\hline $\begin{array}{l}\text { Pulse energy stability (short term }-1 \\
\text { min) }\end{array}$ & $<1 \%$ \\
\hline Pulse energy stability (long term $-1 \mathrm{hr}$ ) & $<5 \%$ \\
\hline Optical Output & $\begin{array}{l}\text { Free space, } \mathrm{PM}, \sim 100 \mu \mathrm{rad} \text { divergence, beams co-aligned to better } \\
\text { than } \sim 20 \mu \mathrm{rad}\end{array}$ \\
\hline Beam quality & $\mathrm{M}^{2}<1.5$ per channel \\
\hline Polarization Extinction Ratio (PER) & $20 \mathrm{~dB}$ \\
\hline
\end{tabular}

The overall architecture is comprised of 3 modules: a seed laser module, a pre-amplifier module and eight parallel power amplifier modules. The seed module is a diode source producing wavelength-stepped, constant-wave (CW) output. The preamplifier module consists of multiple erbium-doped Fiber amplifier (EDFA) stages, amplitude modulation and a signal divider that splits the signal into 8 different output fibers. Each of the preamplifier output fibers feeds a power amplifier module. We split the signal into parallel channels because the fiber power amplifiers' peak power (and therefore pulse energy) is limited by stimulated Brillouin scattering (SBS). The output of the power amplifier modules is incoherently combined by co-aligning the collimated output beams, which will overlap in the far-field and achieve the required pulse energy at the target.

The seed laser module is a continuous wave (CW), wavelength-tuned and locked diode laser [7, 8]. We lock a DFB diode laser to the absorption peak of a Herriot gas cell filled with $\mathrm{CO}_{2}$. This is the master laser and is our absolute wavelength reference. The slave laser is a DBR laser and is dynamically offset-locked to the master. The master and slave laser outputs are fiber combined and the difference in their frequencies creates a beat note. By monitoring the beat note frequency and using that signal in a feedback loop, the slave has a programmable offset in wavelength from the master. It takes less than $40 \mu \mathrm{s}$ to tune from one locked wavelength to the next and the locking has absolute accuracy of $<3 \mathrm{MHz}$. Using this technique we achieve unambiguous spectroscopic knowledge. 


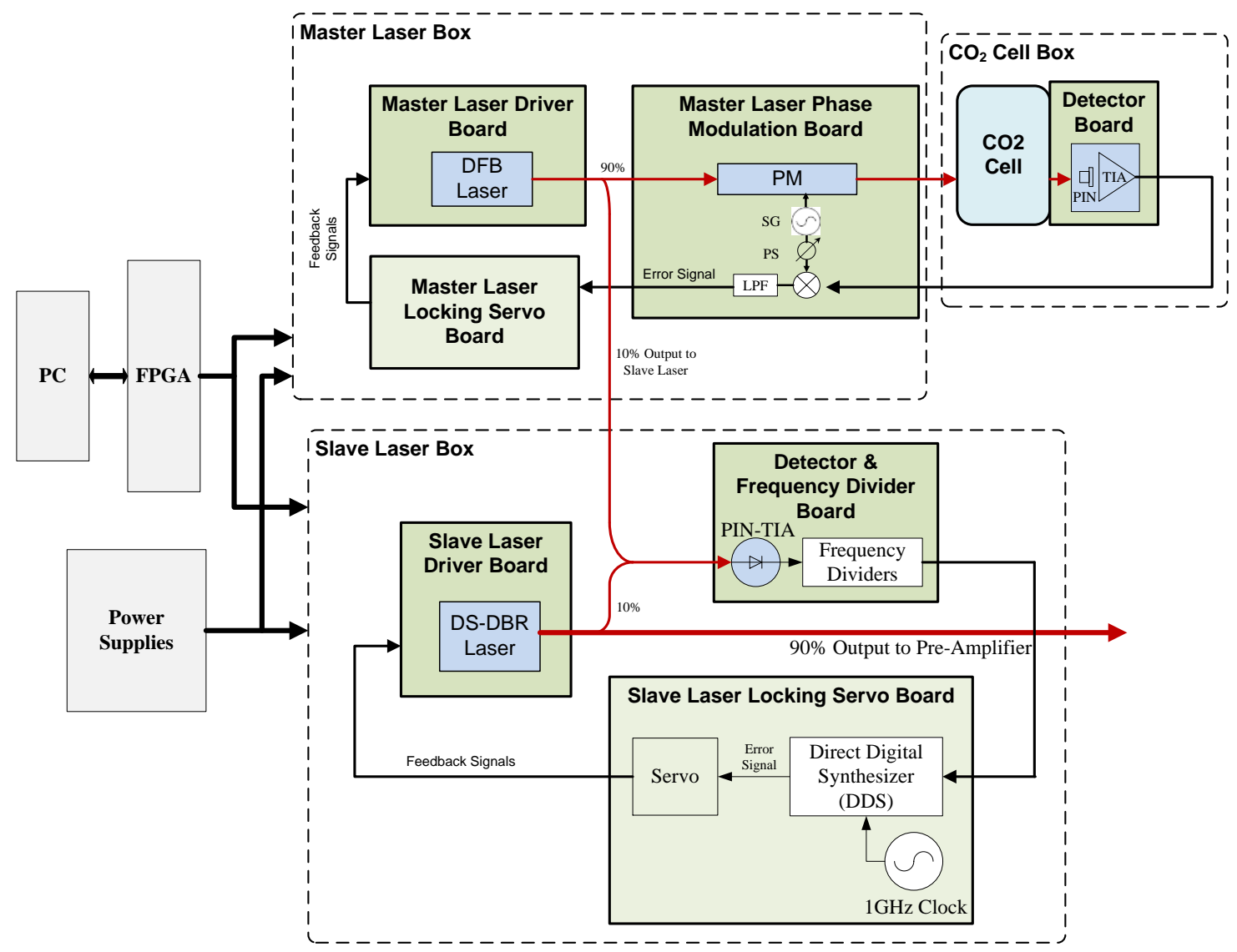

Figure 2. Diagram of the seed laser architecture. Figure shows a master laser, which is locked to a $\mathrm{CO}_{2}$ gas cell and becomes an absolute wavelength reference for the rest of the system. The slave laser is dynamically offset locked from the master. From this configuration we get a frequency-stepped laser that is locked at each required absolute frequency.

DFB - distributed feedback laser; PM - phase modulator; PIN - PIN photo detector; TIA - transimpedance amplifier; PS phase shifter; SG - signal generator; LPF - low-pass filter; FPGA - field-programmable gate array; TEC - thermoelectric cooler. DDS - direct digital synthesizer; DS-DBR - digital supermode distributed Bragg reflector laser;

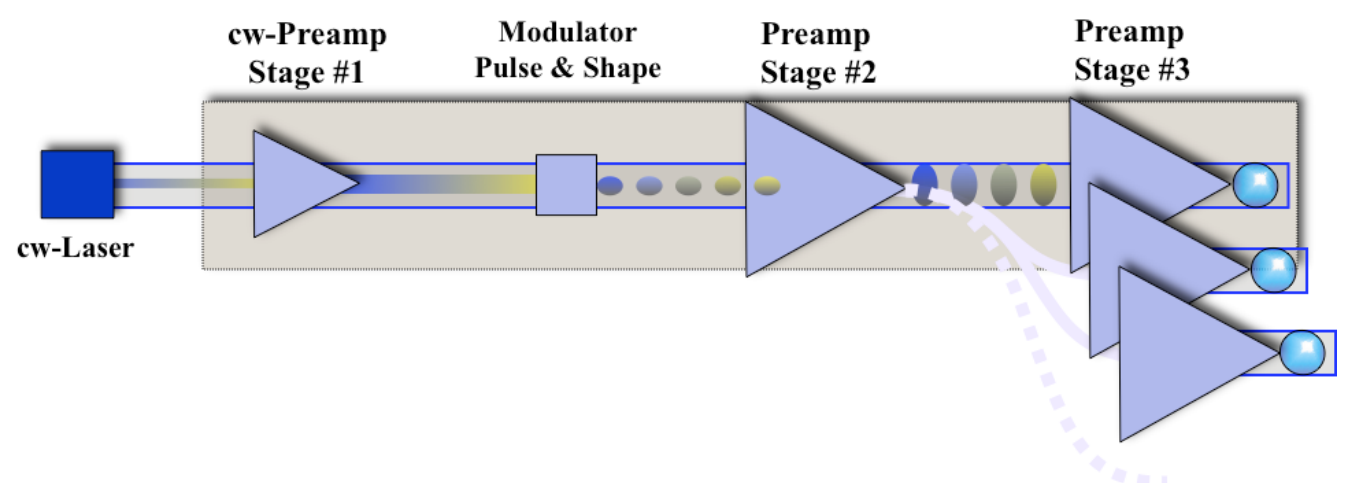

Figure 3. Diagram of the preamplifier module shows multiple amplification stages, modulation and split output signals which are input into the power amplifier modules.

The output of the seed module goes to the pre-amplifier module, which modulates, amplifies and splits the signal. The CW output from the seed is only $\sim 10 \mathrm{~mW}$ so the signal is first amplified up to $100 \mathrm{~mW}$ using a small core EDFA. Due to the requirement for linearly polarized output, all the fibers used in this transmitter are polarization maintaining (PM). The 
signal is then modulated using a Mach-Zehnder modulator (MZM) to carve out $1 \mu$ s wide pulses at a $7.5 \mathrm{kHz}$ repetition rate. The modulation must also shape the pulse to compensate for gain steepening in subsequent amplification. The signal is then further amplified with subsequent EDFAs and filtered to minimize amplified spontaneous emission (ASE) to keep the power in the pulses. The signal is then split into 8 output fibers, each feeding a power amplifier module.

The power amplifier modules further amplify the signal and are then collimated and incoherently combined using freespace optical co-alignment. The baseline is for eight parallel power amplifier modules to reach the needed pulse energy but this will be refined as the performance and margins of the amplifiers are better understood. We are working on two power amplifier architectures: the first is being developed at Fibertek, Inc. and uses commercial Nufern large mode area (LMA) fiber and the second is being developed at OFS laboratories and uses a custom designed PM very large mode area (VLMA) fiber. Option 1 employs commercial Er-Yb-doped PM fiber with a $25-\mu \mathrm{m}$ diameter core manufactured by Nufern. The amplifier is cladding-pumped with $976 \mathrm{~nm}$ diodes. We have developed a multistage amplifier module that has so far demonstrated an SBS-limited peak optical power of $500 \mathrm{~W}$ with a spectral linewidth of $100 \mathrm{MHz}$ at $1572.3 \mathrm{~nm}$. Higher peak powers are achievable at wavelengths centered on the erbium emission peak but we are optimizing performance for this application. Option 2 employs an Er-doped PM VLMA fiber with a 50- $\mu$ m diameter core manufactured by OFS Laboratories. This is not a single mode fiber but single mode performance can be attained reliably if the right launch conditions are achieved and maintained. The amplifier is core-pumped with a $1480 \mathrm{~nm}$ Raman fiber laser. We have developed a single stage amplifier module that has so far demonstrated an SBS-limited peak optical power of $400 \mathrm{~W}$ with a transform-limited linewidth centered at $1572.3 \mathrm{~nm}$. Given the likely increase in SBS threshold if the linewidth were broadened to $100 \mathrm{MHz}$, this means the two approaches currently have very similar performance. A $500 \mathrm{~W}$ peak power, $1 \mu$ s rectangular pulse will have $0.5 \mathrm{~mJ}$ pulse energy. With the desired $\sim 20 \%$ derating, each power amplifier module can be operated at $0.4 \mathrm{~mJ}$ and the combined energy of 8 modules should yield the goal of $3.2 \mathrm{~mJ}$. This allows room for beam combination losses to achieve the overall objective with margin.

To date both approaches show promise for meeting all performance objectives of the laser transmitter. Additional optimization involving SBS threshold, wall-plug efficiency, ASE and fiber length still need to be completed. Pulse shaping tailored to the subsequent amplifiers needs to be completed to achieve a rectangular final output pulse. We will also develop a ruggedized, compact package that will survive our environmental requirements of vibration and vacuum testing as well as radiation tolerance.

\section{ACKNOWLEDEMENT}

The authors would like to acknowledge the support from NASA's Earth Science Technology Office (ESTO) under an Advanced Technology Initiative (ATI) and NASA's Goddard Space Flight Center under an Internal Research and Development (IRAD) award.

\section{REFERENCES}

1. Jeffrey R. Chen, et al., "Error reduction in retrievals of atmospheric species from symmetrically measured lidar sounding absorption spectra," Opt. Express 22, 26055-26075 (2014)

2. NASA ASCENDS Mission Science Definition and Planning Workshop Report, Available from: http://cce.nasa.gov/ascends/12-30-08\%20ASCENDS_Workshop_Report\%20clean.pdf, 2008.

3. Abshire, J.B. et al., "A lidar approach to measure $\mathrm{CO}_{2}$ concentrations from space for the ASCENDS Mission," Proc. SPIE 7832, paper 78320D, November 2010, doi:10.1117/12.868567.

4. Doruk E., et al., "Highly reliable and efficient $1.5 \mu \mathrm{m}$-fiber-MOPA-based, high-power laser transmitter for space communication," Proc. SPIE 9081, Laser Technology for Defense and Security X, 90810 G (9 June 2014); doi: $10.1117 / 12.2050496$.

5. Wei Lu; Doruk Engin; Mark Storm; Shantanu Gupta, "High-power, narrow linewidth $1.5-\mu \mathrm{m}$ fiber amplifier lidar transmitter for atmospheric CO2 detection," Proc. SPIE 8961, Fiber Lasers XI: Technology, Systems, and Applications, 89610C (March 7, 2014); doi:10.1117/12.2041204

6. J.C. Jasapara, et al. "Diffraction-Limited Fundamental Mode Operation of Core-Pumped Very-Large-Mode-Area Er Fiber Amplifiers" IEEE Jour. of Sel. Topics. in Quant. Electron. 15, pp 3-11 (2009).

7. Numata, K., et al., "Frequency stabilization of distributed-feedback laser diodes at $1572 \mathrm{~nm}$ for lidar measurements of atmospheric carbon dioxide," Appl. Opt., 50, 1047-1056 (2011).

8. Numata, K., et al., "Precision and fast wavelength tuning of a dynamically phase-locked widely-tunable laser," Opt. Express, 20, 14234-14243 (2012). 